\title{
A Diophantine equation about polygonal numbers
}

\section{Yangcheng Li}

\author{
School of Mathematics and Statistics, Changsha University of Science and Technology, \\ Changsha, 410114, People's Republic of China \\ e-mail: liyangchengmlx@163.com
}

Received: 8 July 2020

Revised: 15 August 2021

Accepted: 27 August 2021

Abstract: It is well known that the number $P_{k}(x)=\frac{x((k-2)(x-1)+2)}{2}$ is called the $x$-th $k$-gonal number, where $x \geq 1, k \geq 3$. Many Diophantine equations about polygonal numbers have been studied. By the theory of Pell equation, we show that if $G(k-2)\left(A(p-2) a^{2}+2 C a b+B(q-2) b^{2}\right)$ is a positive integer but not a perfect square, $(2 A(p-2) \alpha-(p-4) A+2 C \beta+2 D) a+$ $(2 B(q-2) \beta-(q-4) B+2 C \alpha+2 E) b>0,2 G(k-2) \gamma-(k-4) G+2 H>0$ and the Diophantine equation

$$
A P_{p}(x)+B P_{q}(y)+C x y+D x+E y+F=G P_{k}(z)+H z
$$

has a nonnegative integer solution $(\alpha, \beta, \gamma)$, then it has infinitely many positive integer solutions of the form $(a t+\alpha, b t+\beta, z)$, where $p, q, k \geq 3$ and $p, q, k, a, b, t, A, B, G \in \mathbb{Z}^{+}, C, D, E, F, H \in \mathbb{Z}$. Keywords: Polygonal number, Diophantine equation, Pell equation, Positive integer solution. 2020 Mathematics Subject Classification: 11D09, $11 \mathrm{D} 72$.

\section{Introduction}

A polygonal number [3] is a positive number, corresponding to an arrangement of points on the plane, which forms a regular polygon. The $x$-th $k$-gonal number [3, p. 5] is

$$
P_{k}(x)=\frac{x((k-2)(x-1)+2)}{2},
$$

where $x \geq 1, k \geq 3$. There are many papers about the polygonal numbers and many properties of them have been studied, we can refer to the first chapter of [4] and D3 of [6].

Several authors investigated the question of when a linear combination of two polygonal numbers is a perfect square. Such as M. H. Le [10] $\left(1+9 P_{3}(n)=z^{2}\right.$, proposed by M. Bencze [1]); 
X. G. Guan [5] $\left(1+\frac{8 s^{2}}{s^{2}-1} P_{3}(n)=z^{2}\right)$; M. J. Hu [8] $\left(1+n P_{3}(y-1)=z^{2}\right)$; J. Y. Peng [12] $\left(1+n P_{3}(y-1)=z^{2}\right.$ and $\left.m P_{3}(x-1)+n P_{3}(y-1)=z^{2}\right)$; M. Jiang and Y. C. Li [9] $\left(1+n P_{k}(y)=z^{2}\right.$ and $\left.m P_{k}(x)+n P_{k}(y)=z^{2}\right)$; Y. C. Li [11] $\left(m P_{p}(x)+n P_{q}(y)=z^{2}\right)$.

For the Diophantine equation

$$
P_{3}(a)+P_{3}(b)=P_{3}(c),
$$

K. R. S. Sastry [14] studied the problem: given a natural number $N$, determine the number $T(N)$ of Eq. (1.1) such that $a=N$.

A. Hamtat and D. Behloul [7] proved that all nonnegative integer solutions of Eq. (1.1) are given by

$$
a=m n, \quad b=\frac{n q-m p-1}{2}, \quad c=\frac{n q+m p-1}{2},
$$

where $p q-m n=1, n q-m p-1 \in 2 \mathbb{N}$ and $m, n, p, q \in \mathbb{N}$.

K. R. S. Sastry [15] investigated the positive integer solutions of the Diophantine equation

$$
P_{n}(a)+P_{n}(b)=P_{n}(c), \quad n \geq 3 .
$$

E. Scheffold [16] gave a parametric representation for Eq. (1.2), i.e.,

$$
a=(n-2) r+t, \quad b=(n-2) r+s, \quad c=(n-2) r+s+t,
$$

where $n \geq 3, r, s, t$ are natural numbers such that

$$
r\left((n-2)^{2} r-(n-4)\right)=2 s t .
$$

H. Cohen [2, Corollary 6.3.6.] introduced the general solutions of the Diophantine equation

$$
A x^{2}+B y^{2}=C z^{2}
$$

i.e., "assume that $A B C \neq 0$, let $\left(x_{0}, y_{0}, z_{0}\right)$ be a particular nontrivial solution of $A x^{2}+B y^{2}=C z^{2}$, and assume that $z_{0} \neq 0$. The general solution in rational numbers to the equation is given by

$$
\left\{\begin{array}{l}
x=d\left(x_{0}\left(A s^{2}-B t^{2}\right)+2 y_{0} B s t\right) \\
y=d\left(2 x_{0} A s t-y_{0}\left(A s^{2}-B t^{2}\right)\right) \\
z=d z_{0}\left(A s^{2}+B t^{2}\right)
\end{array}\right.
$$

where $d \in \mathbb{Q}, s, t \in \mathbb{Z}$, and $\operatorname{gcd}(s, t)=1$."

J. Pla [13] investigated some subsets of the rational solutions of the Diophantine equation

$$
a X^{2}+b X Y+c Y^{2}=d Z^{2},
$$

where $a, b, c, d \in \mathbb{Z}$ and $\operatorname{gcd}(a, b, c)=1$.

\section{Main result}

We consider the polygonal numbers satisfying the Diophantine equation

$$
A P_{p}(x)+B P_{q}(y)+C x y+D x+E y+F=G P_{k}(z)+H z,
$$

where $p, q, k \geq 3, p, q, k, A, B, G \in \mathbb{Z}^{+}, C, D, E, F, H \in \mathbb{Z}$. By the theory of Pell equation, we have the following theorem. 
Theorem 2.1. If $G(k-2)\left(A(p-2) a^{2}+2 C a b+B(q-2) b^{2}\right)$ is a positive integer but not a perfect square, $(2 A(p-2) \alpha-(p-4) A+2 C \beta+2 D) a+(2 B(q-2) \beta-(q-4) B+2 C \alpha+2 E) b>0$, $2 G(k-2) \gamma-(k-4) G+2 H>0$ and $E q .(2.1)$ has a nonnegative integer solution $(\alpha, \beta, \gamma)$, then it has infinitely many positive integer solutions of the form $(a t+\alpha, b t+\beta, z)$, where $t, z$ are given by the recurrence relation (3.7) and $p, q, k \geq 3, p, q, k, a, b, A, B, G \in \mathbb{Z}^{+}, C, D, E, F, H \in \mathbb{Z}$.

\section{Proof of the Theorem}

Proof of Theorem 2.1. Suppose $(\alpha, \beta, \gamma)$ is a nonnegative integer solution of Eq. (2.1), i.e.,

$$
A P_{p}(\alpha)+B P_{q}(\beta)+C \alpha \beta+D \alpha+E \beta+F=G P_{k}(\gamma)+H \gamma .
$$

Let $x=a t+\alpha, y=b t+\beta$, then Eq. (2.1) is equivalent to

$$
\varphi(a, b) t^{2}+\phi(a, b) t+\psi(a, b)=G z((z-1)(k-2)+2)+2 H z,
$$

where

$$
\begin{aligned}
\varphi(a, b)= & A(p-2) a^{2}+2 C a b+B(q-2) b^{2}, \\
\phi(a, b)= & (2 A(p-2) \alpha-(p-4) A+2 C \beta+2 D) a \\
& +(2 B(q-2) \beta-(q-4) B+2 C \alpha+2 E) b, \\
\psi(a, b)= & \alpha((p-2) \alpha-(p-4)) A+\beta((q-2) \beta-(q-4)) B \\
& +2(C \alpha \beta+D \alpha+E \beta+F) .
\end{aligned}
$$

Solving it for $z$, we have

$$
z=\frac{G(k-4)-2 H+\sqrt{\Delta}}{2 G(k-2)}
$$

where

$$
\Delta=4 G(k-2)\left(\varphi(a, b) t^{2}+\phi(a, b) t+\psi(a, b)\right)+((k-4) G-2 H)^{2} .
$$

It is necessary to take $\Delta=w^{2}$. Let

$$
X=G(k-2)(2 \varphi(a, b) t+\phi(a, b)), \quad Z=w,
$$

we obtain the Pell equation

$$
X^{2}-G(k-2) \varphi(a, b) Z^{2}=(G(k-2) \phi(a, b))^{2}-G(k-2) \varphi(a, b) L^{2},
$$

where

$$
L=2 G(k-2) \gamma-(k-4) G+2 H .
$$

If $\phi(a, b)>0$ and $L>0$, Eq. (3.3) has a positive integer solution

$$
\left(X_{0}, Z_{0}\right)=(G(k-2) \phi(a, b), L) .
$$

By the theory of Pell equation, if $G(k-2) \varphi(a, b)$ is a positive integer but not a perfect square, the Pell equation

$$
X^{2}-G(k-2) \varphi(a, b) Z^{2}=1
$$

has infinitely many positive integer solutions. Let $(u, v)$ be the least positive integer solution of Eq. (3.4). It is easy to provide infinitely many positive integer solutions of Eq. (3.3) by the formula 


$$
\begin{aligned}
& X_{s}+Z_{s} \sqrt{G(k-2) \varphi(a, b)} \\
& =\left(X_{0}+Z_{0} \sqrt{G(k-2) \varphi(a, b)}\right)(u+v \sqrt{G(k-2) \varphi(a, b)})^{s}, s \geq 0 .
\end{aligned}
$$

Thus,

$$
\left\{\begin{array}{l}
X_{s+1}=2 u X_{s}-X_{s-1} \\
Z_{s+1}=2 u Z_{s}-Z_{s-1}
\end{array}\right.
$$

where

$$
\begin{aligned}
X_{0} & =G(k-2) \phi(a, b), & X_{1} & =G(k-2)(L v \varphi(a, b)+\phi(a, b) u), \\
Z_{0} & =L, & Z_{1} & =L u+G v(k-2) \phi(a, b) .
\end{aligned}
$$

Using the recurrence relations of $X_{s}$ and $Z_{s}$ twice, we get

$$
\left\{\begin{array}{l}
X_{2 s+2}=2\left(2 u^{2}-1\right) X_{2 s}-X_{2 s-2}, \\
Z_{2 s+2}=2\left(2 u^{2}-1\right) Z_{2 s}-Z_{2 s-2}
\end{array}\right.
$$

where

$$
\begin{aligned}
& X_{0}=G(k-2) \phi(a, b), \\
& X_{2}=G(k-2)\left(\phi(a, b) u^{2}+2 L \varphi(a, b) u v+G(k-2) \varphi(a, b) \phi(a, b) v^{2}\right), \\
& Z_{0}=L, \\
& Z_{2}=L u^{2}+2 G(k-2) \phi(a, b) u v+G L(k-2) \varphi(a, b) v^{2} .
\end{aligned}
$$

It is easy to prove that

$$
\begin{aligned}
X_{2 s} & \equiv G(k-2) \phi(a, b) \\
Z_{2 s} \equiv 2 H-G(k-4) & (\bmod 2 G(k-2) \varphi(a, b)),
\end{aligned}
$$

where $s \geq 0$.

By (3.1) and (3.2), we have

$$
t=\frac{X-G(k-2) \phi(a, b)}{2 G(k-2) \varphi(a, b)}, \quad z=\frac{G(k-4)-2 H+Z}{2 G(k-2)} .
$$

Substituting (3.6) into (3.5), we obtain

$$
\left\{\begin{array}{l}
t_{2 s+2}=2\left(2 u^{2}-1\right) t_{2 s}-t_{2 s-2}+2 G(k-2) \phi(a, b) v^{2} \\
z_{2 s+2}=2\left(2 u^{2}-1\right) z_{2 s}-z_{2 s-2}+2((k-2) G-2 H) \varphi(a, b) v^{2},
\end{array}\right.
$$

where

$$
\begin{aligned}
& t_{0}=0, \quad t_{2}=(L u+G v(k-2) \phi(a, b)) v, \\
& z_{0}=\gamma, \quad z_{2}=L \varphi(a, b) v^{2}+\phi(a, b) u v+\gamma .
\end{aligned}
$$

It follows that $t_{2 s}, z_{2 s} \in \mathbb{Z}^{+}$for all $s>0$.

Therefore, if $X_{0}>0, Z_{0}>0, G(k-2) \varphi(a, b)$ is a positive integer but not a perfect square and Eq. (2.1) has a nonnegative integer solution $(\alpha, \beta, \gamma)$, then we get infinitely many positive integer solutions of the form $(a t+\alpha, b t+\beta, z)$, where $t, z$ given by the recurrence relation (3.7). 
Example 3.1. When $A=B=C=D=E=F=G=H=1, p=3, q=4, k=5$, then Eq. (2.1) becomes

$$
P_{3}(x)+P_{4}(y)+x y+x+y+1=P_{5}(z)+z,
$$

it has a positive integer solution $(6,7,9)$. Let $a=b=1$, then $u=4, v=1$. Hence, Eq. (2.1) has infinitely many positive integer solutions $\left(t_{2 s}+7, t_{2 s}+9, z_{2 s}\right)$, where

$$
\left\{\begin{array}{l}
t_{2 s+2}=62_{2 s}-t_{2 s-2}+426, \quad t_{0}=0, \quad t_{2}=433 \\
z_{2 s+2}=62_{2 s}-z_{2 s-2}+10, \quad z_{0}=9, \quad t_{2}=568
\end{array}\right.
$$

where $s$ is a nonnegative integer.

Remark 3.2. For $D=E=F=H=0$, Eq. (2.1) becomes

$$
A P_{p}(x)+B P_{q}(y)+C x y=G P_{k}(z) .
$$

When $p=q=k=4$, this was investigated by J. Pla [13].

Further, if $C=0$, we have

$$
A P_{p}(x)+B P_{q}(y)=G P_{k}(z) .
$$

Case 1: When $p=q=k=4$, Eq. (3.8) becomes

$$
A x^{2}+B y^{2}=G z^{2} .
$$

This is the case studied by H. Cohen [2, Corollary 6.3.6.].

Case 2: When $A=B=G=1, p=q=k$, we have

$$
P_{k}(x)+P_{k}(y)=P_{k}(z),
$$

this case was investigated by K. R. S. Sastry [15] and E. Scheffold [16]. In particular, when $p=q=k=3$, we get

$$
P_{3}(x)+P_{3}(y)=P_{3}(z),
$$

this case was studied by K. R. S. Sastry [14] and A. Hamtat and D. Behloul [7].

Case 3: When $G=1, k=4$, the conclusion becomes a linear combination of two polygonal numbers is a perfect square (see [1, 5, 8-12]).

Remark 3.3. Using the undetermined coefficient method, we obtain some parametric solutions of Eq. (3.8).

Case 1: $(p, q, k, A, B, G, x, y, z)=\left(3,3,3,1,1,1,(2 r+1) t u s^{2}, 2 r(r+1) t u s^{2}+r\right.$,

$$
\left.\left(2 r^{2}+2 r+1\right) \text { tus }^{2}+r\right) \text {. }
$$

Case 2: $(p, q, k, A, B, G, x, y, z)=\left(3,3,3, \frac{(2 b+1)^{2}-(2 c+1)^{2}}{4}, \frac{(2 a+1)^{2}-(2 b+1)^{2}}{4}, \frac{(2 a+1)^{2}-(2 c+1)^{2}}{4}\right.$,

$$
(2 a+1) t+a,(2 c+1) t+c,(2 b+1) t+b), \text { where } a>b>c .
$$

Case 3: $(p, q, k, A, B, G, x, y, z)=\left(k, k, k, 4(k-2)^{2} t^{2}-1,1,1\right.$, as $+(k-4) t$, as,

$$
t(2(k-2)(k-4) t+2 a(k-2) s-(k-4))) .
$$

Case 4: $(p, q, k, A, B, G, x, y, z)=(k-1, k, k+1,1,1,1,1,2,2)$,

$$
(p, q, k, A, B, G, x, y, z)=(3,4,5,1,1,1, t-1, t, t) .
$$




\section{References}

[1] Bencze, M. (2012). Proposed problem 7508. Octogon Mathematical Magazine, 13(1B), 678.

[2] Cohen, H. (2007). Number Theory, Vol. I: Tools and Diophantine Equations, Graduate Texts in Mathematics.

[3] Deza, E., \& Deza, M. M. (2012). Figurate Numbers, World Scientific.

[4] Dickson, L. E. (1934). History of the Theory of Numbers, Vol. II: Diophantine Analysis, Dover Publications.

[5] Guan, X. G. (2011). The squares with the form $1+\frac{4 n(n+1) s^{2}}{s^{2}-1}$. Natural Science Journal of Ningxia Teachers University, 32(3), 97-107.

[6] Guy, R. K. (2007). Unsolved Problems in Number Theory. Springer-Verlag.

[7] Hamtat, A., \& Behloul, D. (2017). On a Diophantine equation on triangular numbers. Miskolc Mathematical Notes, 18(2), 779-786.

[8] Hu, M. J. (2013). The positive integer solutions of the Diophantine equation $1+n\left(\begin{array}{l}x \\ 2\end{array}\right)=y^{2}$. Journal of Zhejiang International Studies University, 4, 70-76.

[9] Jiang, M., \& Li, Y. C. (2020). The linear combination of two polygonal numbers is a perfect square. Notes on Number Theory and Discrete Mathematics, 26(2), 105-115.

[10] Le, M. H. (2007). The squares with the form $1+\frac{9}{2} n(n+1)$. Natural Science Journal of Hainan University, 25(1), 13-14.

[11] Li, Y. C. (2020). Linear combinations of two polygonal numbers that take infinitely often a square value. Integers, 20, Article \#A100.

[12] Peng, J. Y. (2019). The linear combination of two triangular numbers is a perfect square. Notes on Number Theory and Discrete Mathematics, 25(3), 1-12.

[13] Pla, J. (2014). On some subsets of the rational solutions of the equations $a X^{2}+b X Y+c Y^{2}=$ $d Z^{2}$. The Mathematical Gazette, 98(543), 424-428.

[14] Sastry, K. R. S. (1993). A triangular triangle problem. Crux Mathematicorum, 19(8), 219-221.

[15] Sastry, K. R. S. (1993). Pythagorean triangles of the polygonal numbers. Mathematics and Computer Education Journal, 27(2), 135-142.

[16] Scheffold, E. (2001). Pythagorean triples of polygonal numbers. The American Mathematical Monthly, 108(3), 257-258. 